Contrib. Plasma Phys. 39 (1999) 1, in press

\title{
Ionization Equilibrium and Equation of State of Hydrogen Plasmas in Strong Magnetic Fields
}

\author{
A. Y. Potekhin (A), G. Chabrier (B), Yu. A. Shibanov (A), \\ J. Ventura (C,D)
}

(a) Ioffe Physical-Technical Institute, 194021 St.-Petersburg, Russia

(b) CRAL, Ecole Normale Supérieure de Lyon, 69364 Lyon Cedex 07, France

(c) Department of Physics, University of Crete, 71003 Heraklion, Crete, Greece

(d) Institute of Electronic Structure and Laser, FORTH, 71110 Heraklion, Crete, Greece

\begin{abstract}
We study hydrogen plasmas at magnetic fields $B \sim 10^{12}-10^{13} \mathrm{G}$, densities $\rho \sim 10^{-3}-10^{3} \mathrm{~g} \mathrm{~cm}^{-3}$ and temperatures $T \sim 10^{5.5}-10^{6.5} \mathrm{~K}$, typical of photospheres of middle-aged cooling neutron stars. We construct an analytical free energy model of the partially ionized plasma, including into consideration the decentred atomic states, which arise due to the thermal motion across the strong field. We show that these states, neglected in previous studies, may contribute appreciably into thermodynamics of the outer atmospheric layers at $\rho \lesssim 1 \mathrm{~g} \mathrm{~cm}^{-3}$ and typical $B$ and $T$. We take into account Coulomb non-ideality of the ionized component of the plasma affected by intense magnetic field. Ionization degree, occupancies and equation of state are calculated, and their dependences on the temperature, density and magnetic field are studied.
\end{abstract}

\section{Introduction}

Magnetic fields $B \sim 10^{12}-10^{13} \mathrm{G}$ typical of isolated neutron stars qualitatively modify many physical properties of matter [1, 2]. It was suggested that the outer layers of the neutron stars may be composed of hydrogen at temperatures $T \sim 10^{5.5}-10^{6.5} \mathrm{~K}\|3\|$. Thus the study of hydrogen plasmas at such $B$ and $T$ is of great practical importance for astrophysics. For studying the magnetized matter, Thomas-Fermi-like methods were used starting from 1970 [4] (see ref. [5] for recent results and references). It is well known, however, that they are not well suited for light elements. Here we employ the free-energy minimization method.

The motion of charged particles in a magnetic field is quantized into Landau orbitals. The magnetic field is called strongly quantizing if the free electrons populate mostly the ground Landau level [2]. This occurs when the electron cyclotron energy $\hbar \omega_{c}=\hbar e B /\left(m_{e} c\right)$ (where $\hbar, e, m_{e}$ and $c$ are the Planck constant, electron charge, electron mass and speed of light, respectively) exceeds both the thermal energy $k_{B} T$ and the electron Fermi energy $\epsilon_{F}$ - that is for temperatures $T \ll T_{B}$ and densities $\rho<\rho_{B}$, where

$$
T_{B}=3.16 \times 10^{5} \gamma \mathrm{K}, \quad \rho_{B}=0.809 \gamma^{3 / 2} \mathrm{~g} \mathrm{~cm}^{-3}, \quad \gamma \equiv \frac{\hbar^{3} B}{m_{e}^{2} c e^{3}}=\frac{B}{2.35 \times 10^{9} \mathrm{G}} .
$$

The atom in a strong magnetic field $\gamma \gg 1$ is compressed in the transverse directions to the size of the "magnetic length": $a_{m}=(\hbar c / e B)^{1 / 2}=a_{0} \gamma^{-1 / 2}$, where $a_{0}=\hbar^{2} /\left(m_{e} e^{2}\right)$ is the Bohr radius. The ground-state binding energy grows logarithmically with $B$ and exceeds the ground-state energy of the field-free atom by order of magnitude at $B \sim 10^{12} \mathrm{G}$ [1]. Ionization equilibrium of atoms in strong magnetic fields has been first discussed in ref. [6]. However, that pioneering work neglected modifications of the atomic properties caused by the thermal motion of the atoms across the field. These motional modifications arise from the coupling between the centre-of-mass motion across the field and the relative electron-proton motion. These effects were appreciated by Ventura et al. [7], but quantummechanical calculations of binding energies and wave functions of hydrogen atoms in any states of motion in the strong magnetic fields have been carried out only recently [ 8$]$. 
Lai and Salpeter [9] (see references therein for earlier work) considered the ionization equilibrium of strongly magnetized hydrogen using a crude approximation for binding energies of moving atoms which missed the so-called decentred states with a large electron-proton separation [8]. The same approximation was used in ref. [10], devoted to the low-density equation of state. Here we employ new fitting formulae to atomic energies and sizes 11] based on the previous study 8 , valid for any state of atomic motion. We construct an analytic model of the plasma free energy and derive and solve a generalized Saha equation.

\section{Free Energy Model and Generalized Saha Equation}

We consider a plasma consisting of $N_{e}$ electrons, $N_{p}=N_{e}$ protons, and $N_{H}$ hydrogen atoms in a volume $V$, and write the Helmholtz free energy as $F=F_{\text {id }}+F_{\text {ex }}$, where $F_{\text {id }}=$ $F_{\text {id }}^{(e)}+F_{\text {id }}^{(p)}+F_{\text {id }}^{\text {neu }}$ is the sum of the ideal-gas free energies of the electrons, protons, and neutral species, respectively, and $F_{\text {ex }}$ is the excess free energy.

For the ideal gas of electrons, the pressure and number density are

$$
P_{e}=\frac{k_{B} T}{\pi^{3 / 2} a_{m}^{2} \lambda_{e}} \sum_{N=0}^{\infty} g_{N} I_{1 / 2}\left(\beta \mu_{N}\right), \quad n_{e}=\frac{1}{2 \pi^{3 / 2} a_{m}^{2} \lambda_{e}} \sum_{N=0}^{\infty} g_{N} I_{-1 / 2}\left(\beta \mu_{N}\right),
$$

where $I_{p}(x)=\int_{0}^{\infty} t^{p} \mathrm{~d} t /\left(e^{t-x}+1\right)$ is the Fermi integral, $\mu_{N} \equiv \mu_{e}-N \hbar \omega_{c}, \mu_{e}$ is the chemical potential, $\beta \equiv\left(k_{B} T\right)^{-1}, \lambda_{e} \equiv \hbar \sqrt{2 \pi \beta / m_{e}}, g_{N \geq 1}=2$, and $g_{N=0}=1$. The free energy is given by $F_{\text {id }}^{(e)}=\mu_{e} N_{e}-P_{e} V$, where $\mu_{e}$ is found using an algorithm described in ref. [12].

In the strongly quantizing regime, the Fermi energy is $\epsilon_{F}=2 \pi^{4} \hbar^{2}\left(a_{m}^{2} n_{e}\right)^{2} / m_{e}$, which differs from the non-magnetic case by a factor $(4 / 3)^{2 / 3}\left(\rho / \rho_{B}\right)^{4 / 3}$. Thus the degeneracy is strongly reduced at $\rho \ll \rho_{B}$. Furthermore, in the non-degenerate regime $\left(k_{B} T \gg \epsilon_{F}\right)$, we have $F_{\text {id }}^{(e)}=N_{e} k_{B} T\left[\ln \left(2 \pi a_{m}^{2} \lambda_{e} n_{e}\right)-1\right]$.

For the protons, which are non-degenerate, we have

$$
\beta F_{\text {id }}^{(p)} / N_{p}=\ln \left(2 \pi a_{m}^{2} \lambda_{p} n_{p}\right)+\ln \left[1-\exp \left(-\beta \hbar \omega_{c p}\right)\right]-1,
$$

where $\omega_{c p}=\left(m_{e} / m_{p}\right) \omega_{c}$ is the proton cyclotron frequency. Here, for sake of brevity, we drop the zero-point energy $\frac{1}{2} \hbar \omega_{c p}$ and the spin energy $\pm \frac{1}{4} g_{p} \hbar \omega_{c p}$, where $g_{p}=5.585$ is the proton spin gyromagnetic factor. These terms are the same for free and bound protons. Taking them into account yields an additive contribution: $\Delta F=N_{0}\left\{\hbar \omega_{c p} / 2-\right.$ $\left.k_{B} T \ln \left[2 \cosh \left(\beta g_{p} \hbar \omega_{c p} / 4\right)\right]\right\}$, where $N_{0}$ is the total number of protons (free and bound). $\Delta F$ does not affect ionization equilibrium and pressure.

For the excess free energy of the ionized component, a general fitting formula in the non-magnetic case is given in ref. [13]. It is known that thermodynamics of classical Coulomb plasmas is not affected by the magnetic field, which, however, affects the quantummechanical contributions to $F_{\text {ex }}$. These effects have been studied only in the low-temperature or low-density regimes (e.g., ref. [10] and references therein). Here we use a scaling $\left(r_{s}^{\text {eff }}=\right.$ $\left.s r_{s}\right)$ of the density parameter $r_{s}=\left(4 \pi n_{e} a_{0}^{3} / 3\right)^{-1 / 3}$ at a fixed Coulomb parameter $\Gamma=$ $\beta e^{2} /\left(a_{0} r_{s}\right)$ in the formulae of ref. [13]. The scaling is devised so as to reproduce the lowdensity, high-temperature results presented in ref. 10], as well as other known limiting cases. For the contribution of electron-electron and electron-ion interactions in $F_{\text {ex }}$, the scaling factors are $s_{e e}=\left(1+\theta_{m} / \theta_{0}\right) /\left[1+\left(\theta_{m} / \theta_{0}\right) \exp \left(-\theta_{m}^{-1}\right) f_{1}\right]$ and $s_{i e}=1 / f_{2}^{2}$, where $\theta_{0}=2(9 \pi / 4)^{-2 / 3} r_{s} / \Gamma$ and $\theta_{m}=8 \gamma^{2} r_{s}^{5} /\left(9 \pi^{2} \Gamma\right)$ are the non-magnetic and magnetic degeneracy parameters, respectively, and the factors $f_{1}$ and $f_{2}$ (depending on $\beta \hbar \omega_{c}$ ) are given in ref. 10.

The ideal-gas contribution of the magnetized atoms reads

$$
\beta F_{\mathrm{id}}^{(H)}=\sum_{s \nu} \int \mathrm{d}^{2} K_{\perp} N_{s \nu}\left(K_{\perp}\right)\left\{\ln \left[n_{H} \lambda_{H}^{3} w_{s \nu}\left(K_{\perp}\right) / Z_{w}\right]-1\right\}
$$


where $s$ and $\nu$ relate to electronic excitations, $N_{s \nu}=\left(\lambda_{H} / 2 \pi \hbar\right)^{2} N_{H} w_{s \nu} \mathrm{e}^{\beta \chi_{s \nu}} / Z_{w}$ are the atomic occupancies per unit phase space of the transverse component $K_{\perp}$ of the pseudomomentum $\mathbf{K}$ which characterizes the atomic motion in the magnetic field, $w_{s \nu}\left(K_{\perp}\right)$ and $\chi_{s \nu}\left(K_{\perp}\right)$ are the occupation probabilities and binding energies of the moving atom, and $Z_{w}=\left(\lambda_{H} / 2 \pi \hbar\right)^{2} \sum_{s \nu} \int \mathrm{d}^{2} K_{\perp} w_{s \nu}\left(K_{\perp}\right) \exp \left[\beta \chi_{s \nu}\left(K_{\perp}\right)\right]$ is the internal partition function.

The contribution of atoms in the nonideal part $F_{\text {ex }}$ of the free energy is calculated in the hard-sphere approximation using the van der Waals one-fluid model by analogy with ref. 14. Its straightforward generalization to the magnetic case involves the composite quantum number $\kappa=\left(s \nu K_{\perp}\right)$, so that $\Sigma_{\kappa}$ includes now integration over $K_{\perp}$. The hardsphere diameters are set equal to the effective atomic sizes $l_{\kappa}$ given in [11]. The occupation probabilities are then given by formulae derived in ref. [14], extended to the magnetic case.

Our model is valid as long as the formation of molecules may be neglected. In order to quantify the range of validity, we estimate the abundance of $\mathrm{H}_{2}$ molecules following ref. [9], but with inclusion of the non-ideal effects.

Minimization of the free energy yields the ionization equilibrium (generalized Saha) equation:

$$
n_{H}=n_{p} n_{e}\left(\lambda_{p} \lambda_{e} / \lambda_{H}^{3}\right)\left(2 \pi a_{m}^{2}\right)^{2}\left[1-\exp \left(-\beta \hbar \omega_{c p}\right)\right] Z_{w} \exp (\Lambda),
$$

where $\Lambda=\beta \mu_{e}-\ln \left(2 \pi a_{m}^{2} \lambda_{e} n_{e}\right)+\beta \partial \mu_{e} / \partial \ln n_{e}-\partial P_{e} / \partial n_{e}$ takes into account effects of electron degeneracy and population of excited Landau levels.

\section{Results and Discussion}

Figure 1 s shows selected results obtained for $B=10^{12} \mathrm{G}$. The left panel shows the neutral fraction of atoms $f_{H}=N_{H} / N_{0}$ and molecules $f_{H 2}=2 N_{H 2} / N_{0}$ at $T=10^{6} \mathrm{~K}$. For comparison, we plot the fraction of atoms in the centred states, $f_{H}$ according to ref. [9] and $f_{H}$ in the non-magnetic case. Long dashes display the fraction of atoms that satisfy the Inglis-Teller (IT) criterion and thus can be identified in optical spectra of the plasma. The IT fraction is estimated according to the formula $n_{\kappa}^{\mathrm{IT}} \sim n_{\kappa} \exp \left[-n_{p}\left(4 l_{\kappa}\right)^{3}\right]$ (cf. Eq. (31) of ref. [14]). We can see that (a) the strong magnetic field increases the non-ionized fraction and shifts the region of pressure ionization to much higher $\rho$ (compare the solid line and triangles in the left panel of Fig. 1), (b) the approximation of ref. [9] reproduces only the abundance of the centred atoms at low density and fails at high density where the pressureionization effects are important, and (c) at the low density, the decentred atomic states are significantly populated.

The right panel demonstrates the equation of state, which is seen to be much softer than (a) in the non-magnetic case (mainly because of the electron degeneracy "taken away" by the strongly quantizing field, but also due to the increased neutral fraction) and (b) in the magnetic but ideal proton-electron plasma (because the Coulomb interactions yield negative contribution to the pressure).

The obtained results are used for modelling neutron-star atmospheres. In particular, the IT fraction of atoms, multiplied by the absorption cross sections calculated in ref. 15], determines an atomic contribution to atmospheric opacities. Preliminary calculations of the opacities, carried out with a simplified $F_{\text {ex }}$, were presented in ref. [16]. The more elaborated model of the plasma described here confirms qualitative results of that work. An important conclusion is that the bound species contribute significantly to the absorption at $B=10^{12}-10^{13} \mathrm{G}$, even at relatively high $T \sim 10^{6} \mathrm{~K}$.

\section{Acknowledgements}

The work was supported in part by the grants RFBR 96-02-16870a, DFG-RFBR 96-02-00177G, and INTAS 96-0542. A.Y.P. acknowlegdes a visiting professorship in the theoretical astrophysics group of the Ecole Normale Supérieure de Lyon. The participation of A.Y.P. in the PNP-9 Workshop has been supported in part by Deutsche Forschungsgemeinschaft and Russian Foundation for Basic Research. 

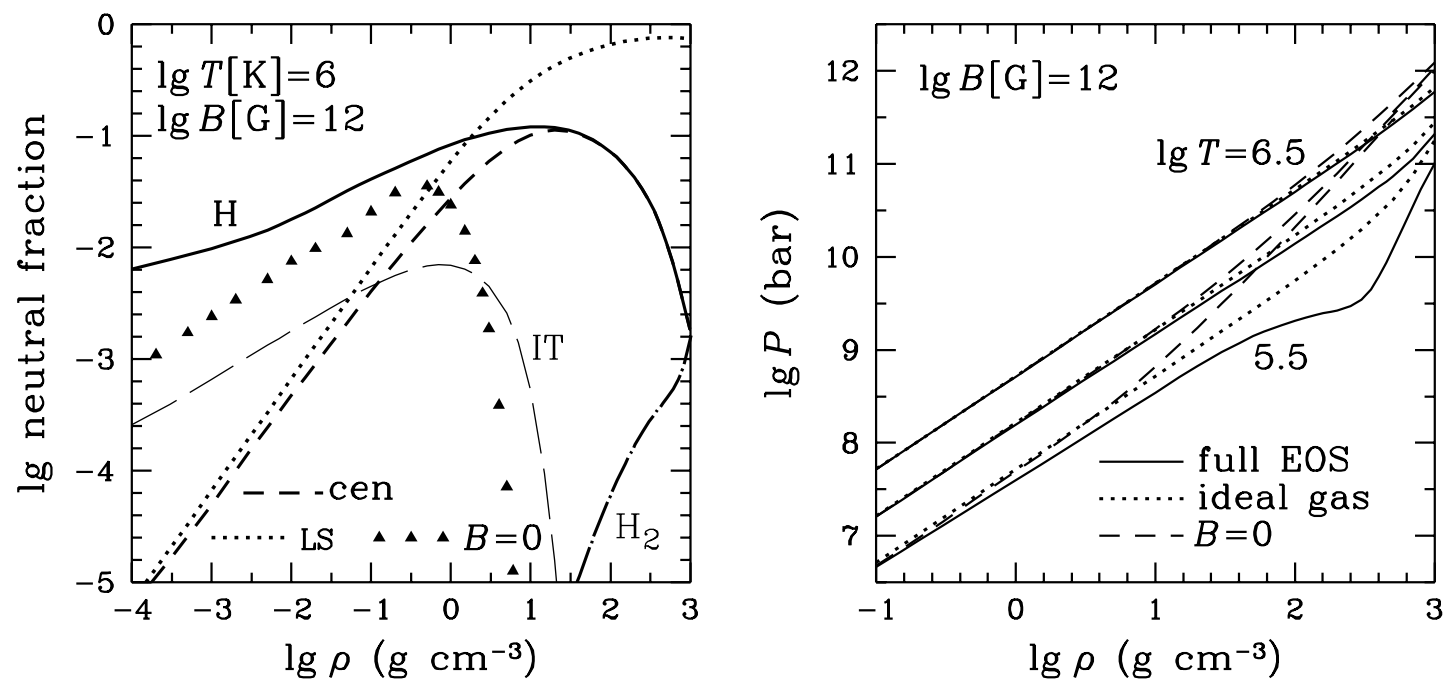

Figure 1: Left panel: Non-ionized fraction of atoms in any states (solid line), atoms in the centred states (short-dashed line), molecules (dash-dot line), and the weakly perturbed atoms contributing to the optics (long-dashed line), compared with the non-magnetic case (triangles) and the approximation [9] (dotted line). Right panel: Pressure isotherms of magnetized hydrogen plasma (solid lines) at $\lg T[\mathrm{~K}]=5.5,6.0$ and 6.5, compared with the non-magnetic case (dashed lines) and with the ideal magnetized plasma (dotted lines).

\section{References}

[1] Canuto, V., Ventura, J., Fundam. Cosm. Phys. 2(1977)203

[2] Yakovlev, D.G., Kaminker, A.D., in The Equation of State in Astrophysics, Chabrier, G., Schatzman, E. (eds.), Cambridge Univ. Press, Cambridge (1994), p. 214

[3] Potekhin, A.Y., Chabrier, G., Yakovlev, D.G., Astron. Astrophys. 323(1997)415

[4] Kadomtsev, B. B., Zh. Eksp. Teor. Fiz. 58(1970)1765 [Sov. Phys.-JETP 31(1970)945]

[5] Thorolfsson, A., Rögnvaldsson, Ö. E., Yngvason, J., Gudmundsson, E. H., Astrophys. J. 502(1998)847

[6] Gnedin, Yu.N., Pavlov, G.G., Tsygan, A.I., Sov. Phys.-JETP 38(1974)903

[7] Ventura, J., Herold, H., Ruder, H., Geyer, F., Astron. Astrophys. 261(1992)235

[8] Potekhin, A.Y., J. Phys. B 27(1994)1073

[9] Lai, D., Salpeter, E.E., Astrophys. J. 491(1997)270

[10] Steinberg, M., Ortner, J., Ebeling, W., Phys. Rev. A 58(1998)3806

[11] Potekhin, A.Y., J. Phys. B 31(1998)49

[12] Potekhin, A.Y., Yakovlev, D.G., Astron. Astrophys. 314(1996)341

[13] Chabrier, G., Potekhin, A.Y., Phys. Rev. E 58(1998)4941

[14] Potekhin, A.Y., Phys. Plasmas 3(1996)4156

[15] Potekhin, A.Y., Pavlov, G.G., Astrophys. J. 483(1997)414

[16] Potekhin, A.Y., Shibanov, Yu.A., Ventura, J., in Neutron Stars and Pulsars, Shibazaki, N., Kawai, N., Shibata, S., Kifune, T. (eds.), Universal Academy Press, Tokyo (1998), p.161

Received 1 October 1998 